\title{
Neural encoding principles in face perception revealed using non-primate models
}

\author{
Keith M Kendrick $^{1}$ and Jianfeng Feng ${ }^{2}$
}

\author{
1. Cognitive and Systems Neuroscience Group, The Babraham Institute, Cambridge CB22 3AT, \\ $U K$. \\ 2. Department of Computer Science, Warwick University, Coventry CV4 7AL UK
}

\begin{abstract}
Specialised neural systems for encoding faces and face emotion cues have been found in sheep which are very similar to those described in human and non-human primates. Sheep exhibit highly sophisticated face identity and face emotion discrimination skills, use configural cues and also have a right hemisphere dominance in face processing. Various neuroanatomical approaches have identified the different brain regions involved and electrophysiological recording methods have established some of the encoding strategies utilised, particularly in the temporal and frontal cortices. Increasingly, these electrophysiological studies are moving into multiarray recording strategies and being used to inform computational and modelling approaches. This chapter will provide an overview of what we know from studies on sheep about the neural substrates involved in face identity and face emotion processing. The main focus though will be on electrophysiological studies which have provided evidence for both high-level sparse encoding and also distributed population encoding as well as some differences in responses in left and right hemispheres. The effects of learning will be discussed together with view-dependent and view-independent and norm-based aspects of encoding as well as mental imagery. The roles of face-stimulus evoked changes in theta and gamma frequency activity will also be discussed and the use of neural network models and simulations to aid functional interpretation of neurophysiological data
\end{abstract}

\section{Introduction}

One of the long held assumptions in the field of face recognition research, namely that specialised processing of face cues was a facet of primate social evolution, was dispelled when we first published electrophysiological findings from sheep inferotemporal cortex reporting the presence of populations of face-sensitive neurons broadly similar to those found in monkeys (Kendrick and Baldwin 1987). From our subsequent detailed behavioural, neuroanatomical mapping and further electrophysiological studies in sheep (Tate et al., 2006) it has become clear that specialised face recognition systems in the brain are likely to have evolved more as a general facet of mammalian social evolution and are probably present in a number or ungulate and other mammalian species with complex social systems and well developed visual acuity. As such, neurophysiological and behavioural studies on sheep face recognition can be considered broadly relevant as a model for helping to understand similar, although undoubtedly more complex, processes in humans.

\section{Face recognition in sheep}

The first behavioral evidence for face recognition in sheep came from observations that maternal sheep found it difficult to recognise their lambs when the latter had their heads artificially colored (Alexander and Shillito-Walser, 1977). Subsequently, using various face discrimination paradigms it has been possible to show that sheep have a highly developed expertise. This was shown initially in a simple two-choice maze where sheep could immediately discriminate between face pictures of individuals with differential attraction (socially familiar vs unfamiliar sheep or sheep vs humans) in order to gain access to the individual whose face they had seen (Kendrick et al., 1995, 1996). Then, using food to reward accurate discrimination between individuals of similar attraction, it was shown both in a two choice maze, or with an 
operant response paradigm (with the sheep indicating choice by pressing one of two panels with its nose), that animals could recognise up to at least 25 pairs of sheep faces simultaneously (Kendrick et al. 2001a) and 4-5 pairs of human faces (Peirce et al. 2001). As with humans, they make use of configural cues for recognising faces, exhibiting both classical inversion effects with both sheep and human faces (Kendrick et al. 1996; Peirce et al. 2001) and the ability to use internal face features for recognition of familiar sheep (Peirce et al. 2000), although not humans (Peirce et al. 2001). Recognition of familiar sheep faces with scrambled internal features is also impaired (Peirce et al. 2001).

Learning to recognise new faces in an experimental context tends to take a minimum of a least 30-40 trials for sheep faces, although learning new associations with faces socially familiar individuals and food rewards can take only 10-20 trials (Kendrick et al. 1996). With human faces novel individuals require on average about 75 trials and learning new reward associations with familiar faces 20-35 trials (Peirce et al. 2001).

Behavioral experiments have also revealed a right hemisphere advantage for face recognition in sheep with the half of the face appearing in the left visual field (from which information is processed by the right brain hemisphere) being utilised most for recognition of familiar sheep faces even under free-viewing circumstances (Peirce et al. 2000).

In terms of visual acuity for face recognition, computer morphing approaches have shown that sheep are able to discriminate between two sheep faces that are only 5-10\% different from one another (Tate et al. 2006). They are also still able to discriminate above chance between faces that are one-third of normal size although accuracy is significantly reduced (Full size $87.6 \pm 1.3 \%$ correct vs one-third size $65.0 \pm 2.5 \%$ correct, paired t-test $\left.\mathrm{t}^{9}=6.46, \mathrm{P}<0.001\right)$. This may also explain why maternal ewes can take several weeks to recognise the faces of their lambs (Kendrick et al. 1996).

Less has been done to establish developmental aspects of face recognition expertise in sheep although lambs are unable to perform even the simplest discrimination of their mother's face from that of another sheep for at least 2-4 weeks after they are born (Kendrick, 1994). We have also had very little success in training sheep in face recognition tasks involving socially unfamiliar animals until they are at least 4-6 months of age. It seems likely therefore that it takes time and experience for a fully mature face recognition system to develop in this species, as it does it humans.

\section{Face attraction and face emotion recognition in sheep}

When sheep are socially isolated they show reduced behavioural and autonomic signs of psychological stress when they are able to view face pictures of individuals of their own breed (da Costa et al. 2004). They also show clear preferences for the faces of socially familiar animals (Kendrick et al. 1995, 1996). Females show marked changes in their relative attraction towards male and female faces as a function of their oestrus cycle (Kendrick et al. 1995) and when they are sexually receptive will exhibit clear preferences for the faces of specific individual males (Fabre-Nys et al. 1997). Cross-fostering studies in both sheep and goats have also found that development of facial preference for one species over the other is determined by the species of the mother who rears them and with whom they have bonded (Kendrick et al. 1998; 2001b).

Sheep have been found to be able to discriminate accurately between both calm and stressed/anxious sheep faces and between human faces that are either smiling or angry. Here it was found that the animals preferred to chose calm or smiling versions of the same sheep and human faces respectively compared with stressed or angry ones (Elliker, 2006; Kendrick, 2008; Tate et al. 2006). This preference for calm as opposed to stressed/anxious faces even takes precedence over the preference for the faces of socially familiar compared to socially unfamiliar individuals (Tate et al. 2006). 
In an operant discrimination experiment on five sheep we systematically varied the appearance of calm and stressed/anxious versions of the same sheep face to try and identify the main features the animals were relying on to discriminate between them. The single most important feature was the positioning of the ears (they tend to be lowered and pointing backwards when the animal is stressed), however some other internal face features such as the eyes and nose may also play some role (Figure 1).

\section{Neural substrates of face recognition in sheep}

A number of studies have been carried out to map the neuroanatomical substrates involved in face recognition is sheep using quantification of expression changes in gene markers of neural activation (c-fos and zif/268). These have shown consistent activation in the medial and posterior inferior and superior temporal cortices, medial prefrontal cortices and basolateral and basomedial amygdala and hippocampus during successful discrimination between or exposure to faces (Broad et al. 2000; da Costa et al. 2004; Ohkura et al. 1997). Face inversion also significantly reduces activation in the right medial inferior temporal cortex and bilaterally in the amygdala and hippocampus. In the temporal (see Figure 2) and frontal cortices and the amygdala activation is stronger in the right than in the left hemisphere (Broad et al. 2000; da Costa et al. 2004). When faces with a particularly strong attraction are shown (oestrus females viewing males or socially isolated animals viewing a familiar breed of sheep) then dopamine reward centres such as the nucleus accumbens are also activated (da Costa et al. 2004; Ohkura et al. 1997).

The area of temporal cortex responding to face stimuli is quite large (at least $1 \mathrm{~cm}^{3}$ ). At this stage it is not clear whether sheep have a similar patch arrangement of face-sensitive regions that have been found in both monkeys and humans (see Freiwald and Tsao, 2009). However, it can been seen from dark field autoradiograms of c-fos and zif/268 mRNA expression changes in Figure 2 that there are distinct clumps of activation hotspots in superior and inferior temporal cortex of sheep when they view faces.

It is clearly difficult to assess the extent to which the same brain regions that become activated during actual conscious perception of faces are also activated when a sheep forms a "mental" image of them, as has been shown in humans (O'Craven and Kanwisher, 2000). However, we have shown that similar c-fos expression changes occur in the region of temporal cortex that responds to faces when maternal ewes hear recordings of the bleats of their absent lambs but not scrambled sequences of these same bleats. The activation levels seen are very similar to those in response to an actual face picture of the lamb (Kendrick et al. 2001c). We have no evidence for vocalisation-specific responses from neurons in this region so this is possibly the result of the animals forming a visual mental image of their lamb's face. This conclusion is further supported by some of the electrophysiological recording findings discussed in a later section.

While we have not investigated changes in molecular markers of neural plasticity during face learning paradigms per se we have found significant increases in mRNA expression of brain derived nerve growth factor (BDNF) in the medial inferior temporal cortex, medial prefrontal cortex, hippocampus and basolateral amygdala of maternal sheep during the period when they learn to recognise their lambs There are also changes in mRNA expression of its receptor, trk- $B$, in the medial inferior temporal cortex at this time (Broad et al. 2002).

\section{Face-specific evoked potentials?}

As in humans (Truett et al. 1999) and monkeys (see Freiwald and Tsao, 2009) we have found some evidence for face-sensitive components of the visual evoked potential recorded by multiple $(n=64)$ electrodes in either right of left inferotemporal cortex. Figure 3 shows averaged evoked potentials following exposure to pairs of faces, inverted faces or non-face objects in a discrimination task. The primary components of the evoked potential are a P120 and an N300 
and the P120 is largest for faces in both animals we have investigated with the profiles for inverted faces and non-face objects looking very similar. In both cases the animals were not able to do better than chance in discriminating between the inverted faces whereas performance was $>80 \%$ correct for normal upright views of the same faces. With the non-face object pairs used the animals also discriminated between them at $>80 \%$ correct. However, we have not investigated this extensively enough, or with single faces, as opposed to face pair stimuli, to conclude categorically that this P120 component is truly face sensitive.

\section{Characteristics of face-sensitive neurons in temporal and frontal cortices}

The first study reporting face-sensitive neurons in the sheep temporal cortex found broadly similar general response properties as had been described in monkeys, namely large receptive fields, short latencies $(80-180 \mathrm{~ms})$ and with a similar population size $(\sim 7 \%)$. Neuronal responses usually did not outlast the period of face presentation and many cells appeared best tuned to frontal views (Kendrick and Baldwin, 1987; Kendrick, 1991). However, an additional observation was that cells responded weakly or not at all to inverted faces. The most important difference with the studies on monkeys was that in the sheep different sub-populations of cells appeared to be categorising for faces with specific behavioural or emotional significance rather than just faces or face features per se. Cells were either optimally tuned to respond to faces of sheep of the same breed, and maximally to social familiar individuals (sheep prefer to be with members of their own breed and form consortships with specific individuals); animals with horns and how large they were (the presence and size of horns provides information both on gender and social dominance) and a final sub-population responded to faces of either dogs or humans (the two main classes of individuals sheep normally perceive as being a potential threat)(Kendrick and Baldwin, 1987). Of these categories the cells responding to horns and their size appeared to be relatively insensitive to identity (human faces with horns were just as effective as sheep or goats with horns) although the horns had to be viewed in the context of a face (Kendrick, 1994). There may well therefore be the same kind of norm-based encoding occurring in the temporal cortex of sheep (i.e. in this case a generic face with horns) as has been reported in monkeys (Leopold et al. 2006).

Faces were not the only body component encoded for my cells in the temporal cortex with a similar proportion of cells being found to be sensitive to the standard bipedal human body shape (even with the head region covered) and which did not respond to humans adopting a quadrupedal posture (Kendrick and Baldwin, 1989). In other studies we have also occasionally found cells highly specifically tuned to specific aggressive body movements in sheep such as foot stamping (Peirce and Kendrick, unpublished observations).

Subsequent single electrode electrophysiological studies in both the medial frontal and temporal cortex regions have shown that cells can often be optimally tuned to the faces of one or two specific familiar sheep and/or humans (Kendrick et al. 2001a; Peirce and Kendrick, 2002). Furthermore, the response latencies of cells are longer the more selectively they are tuned to individual faces, suggesting a degree of hierarchical encoding (Kendrick, 1994; Peirce and Kendrick, 2002).

Face-sensitive cells in both temporal and frontal cortices can either be categorised as view dependent (responding selectively to front or profile views of faces) or view independent (responding equivalently to front and profile views). In the temporal cortex the relative distribution of these responses types is around 50:50 whereas in the frontal cortex it is around 70:30 in favour of the view independent type. Overall the response latencies are faster ( $\sim 50 \mathrm{~ms})$ for the view-dependent cells compared with view independent ones once again implying a greater level of processing in the latter and potentially a degree of hierarchical encoding as well. Not only are the view dependent cells selective for face view but they are also very sensitive to a wide variety of manipulations of facial features and appearance and particularly by inversion. On the other hand the view independent cells show broadly equivalent responses to these 
manipulations and therefore appear relatively insensitive to both view and facial feature content (Tate et al. 2006). This further reinforces the possibility that the view independent cells are involved in higher level encoding of faces than are the view dependent ones. Similar viewindependent cells responding to different views of specific individuals have also been reported in humans (Quian-Quiroga et al. 2005) and monkeys (Perrett et al. 1991). As in sheep, response latencies are longer for these view-independent cells in monkeys than for view dependent ones (Perrett et al. 1991).

In terms of the effects of learning and experience we have been able to show that the broad categories of differential encoding of faces we first reported (Kendrick and Baldwin, 1987) are not rigid. In subsequent single-cell recording studies where the sheep used had been exposed to the same familiar stockman for several years his face, but not those of other humans, was responded to by the majority of cells that encoded faces of socially familiar sheep. There were also a number of cells that responded highly selectively to just his face in the same way as other cells responded highly selectively to the faces of specific, socially familiar sheep (Kendrick et al. 2001a). Indeed, when we compared temporal and frontal cortex single-neuron response profiles across three different groups of sheep that had either had past and current extensive experience of another sheep and this stockman; or only past experience (they had not seen them for a year or more); or no experience of them at all, a number of principles emerged. As would be expected if learning is playing an important role, when the sheep had no experience of these two individuals no cells were found which encoded selectively for their faces and the stockman's face was not responded to by any sheep face selective cells. With the group that had not had experience of the sheep and stockman for a year or more then there were still many cells which responded to both of their faces along with other familiar sheep, whereas there were fewer responding to either of them selectively. This latter finding mirrored behavioural evidence that sheep face can be remembered for up to 2 years or more, and suggests that forgetting the faces of previously familiar individuals involves a progressive loss of selective encoding for them (Kendrick et al. 2001a).

Generally speaking neuronal responses to static faces in sheep inferotemporal and frontal cortex do not outlast the period of presentation. However, we have found that when using video sequences showing the eventual appearance of a familiar individual, that cells which normally only respond during the period of static presentation of its face will start to respond to parts of the video sequence which show that the individual is about to appear, and also when it is expected to appear but has been edited out of the film (Kendrick et al. 2001c). Further, and in support of our c-fos experiments already mentioned above (Kendrick et al. 2001c), we have found evidence for face-sensitive cells in the frontal cortex responding to odours of the same individuals but when face and odour are combined there are no additive effects and very few other cells can be found which respond to odour stimuli alone. Only the view-independent facesensitive cells showed this response to odour stimulation and the same was the case for the cells described above responding to the video sequences. It seems possible therefore that facesensitive cells can respond to mental images of faces in sheep and that it is view-independent type cells which are of most importance in this context. Indeed, it is interesting that in humans view-independent face-sensitive cells in the medial temporal lobe can sometimes also respond to letters spelling an individual's name (Quian-Quiroga et al. 2005). Possibly in such cases the name of a familiar person might evoke transient mental image of their face.

\section{Face processing in left and right temporal cortex}

Despite the fact that sheep, like humans, show a left visual field advantage (right brain hemisphere dominance) for recognising faces (Peirce et al. 2000), and that molecular markers of neural activation are more strongly expressed in the right temporal cortex than in the left after the animal is exposed to face stimuli (Broad et al. 2000; da Costa et al. 2004 - see Figure 2), we have so far been unable to establish any major significant difference in the tuning of cells 
to specific faces or face categories in the left and right temporal cortices (Kendrick and Baldwin, 1987; Peirce and Kendrick, 2002; Kendrick, 2006). A similar absence of inter-hemispheric differences in encoding by face sensitive cells has also been reported in monkeys (Perrett et al. 1988). There was a trend however for

view-dependent cells in the right hemisphere to show more profound reductions in responses to face images where the left visual field half of the face (right half) was blocked out, and no effect whatsoever when the right visual side (left half) was blocked out (Tate et al. 2006).

There are however significant latency differences in the responses of higher order facesensitive cells (those responding to a category of face or to faces of specific individuals) but not for those responding generically to both face and non-face stimuli (Peirce and Kendrick, 2002). With these higher order, face-sensitive cells our single-electrode recordings have found responses in the left temporal cortex are, on average, $~ 50-100 \mathrm{~ms}$ slower in the left hemisphere than in the right. Indeed $\sim 30 \%$ of cells in the left hemisphere had response latencies of $>300 \mathrm{~ms}$ compared with only $\sim 5 \%$ on the right and this is likely beyond the time required to recognise the faces and may instead reflect memory or emotion processing aspects of face perception (Peirce and Kendrick, 2002).

There are always potential sampling error considerations that need to be taken into account with single recording electrode based studies and so more recently we have confirmed these hemispheric latency differences in the temporal cortex using 64-electrode arrays capable of recording from up to around 250 single cells simultaneously. These have shown that overall response latencies in the left hemisphere are on average some 40-80ms slower than those on the right for both cells showing excitatory or inhibitory responses when face discrimination on the basis of identity is required. However, when the basis for the discrimination is the respective emotional cues exhibited on the faces this latency difference disappears (Tate et al. 2006).

More recently we have applied partial Granger causality (Ladroue et al. submitted) to try to establish whether there are causal relationships between processing in the left and right temporal cortex and their directionality. Preliminary evidence using time-series data from local field potentials recorded from up to 60 different electrodes in each hemisphere has shown that following successful learning of a discrimination between two sheep faces to obtain a food reward there is an increased in the number of significant causal relations between electrodes in the right but not the left temporal cortex. Whereas before learning there are more frequent causal influences from the left to the right hemisphere after learning the right hemisphere becomes more dominant in this respect (Figure 4). This suggests that as faces become familiar, and are categorised more at an individual level, then the delayed response times seen in the left hemisphere may be as the result of the left temporal cortex receiving inputs via face processing networks on the right.

\section{Sparse and population-based encoding principles}

The face recognition system brings into sharp focus the ongoing debate as to whether sparse or population based codes or both are operating in the various cortical and sub-cortical networks involved. Only with the development of techniques where activities of multiple individual neurons can be recorded has it been possible to effectively address these different possibilities. In broad agreement with both monkey and human-based studies our single neuron recordings from repeated single-electrode penetrations in sheep temporal and frontal cortices have revealed strong support for sparse encoding strategies being utilised by face-processing networks, with clear examples of hierarchical encoding and high level identity tuning of specific faces by very small numbers of cells rather than, for example, by many cells responding to more simple generic aspects of visual cues associated with faces. There also appears to be a degree of spatial clustering occurring, with cells with similar tuning to face categories or individuals often being encountered adjacent to one other on the same electrode track and distinct hotspots for specific face pairs being seen in multiarray recordings in the temporal cortex (Figure 5). 
However, our multiarray recording experiments also provide compelling evidence that population-based encoding is occurring and it is clear that such population codes can hugely increase the representational capacity of neural networks (Rolls et al. 1997). Activity profiles across recording arrays in the temporal cortex reveal distinct spatially discrete hot spots but also overlapping distributions with different face pairs evoking altered firing of a large proportion of neurons. Findings to date have shown that the array difference (an estimate of the reliability of the representation of a particular face stimulus across an array of neurons) variability in the population firing patterns when repeating stimuli is smallest with simple non-face objects and largest where different face identities are presented. The same animal face exhibiting different emotions (calm vs stressed) is intermediate (Tate et al. 2006). Familiar/learned faces also tend to evoke changes in activity of fewer neurons than unfamiliar ones with the main difference being that fewer cells with excitatory responses are encountered (Tate et al. 2006). There seems therefore to be evidence for sparsening of population encoding as a face becomes familiar coupled with a greater degree of inhibitory activity in the network.

Overall therefore there appears to be both sparse and population-based representation of faces in sheep temporal cortex as in monkeys and humans. In monkey temporal cortex, for example, evidence for population based encoding in face patches has also been found (see Freiwald and Tsao, 2009). A combination of sparse and distributed representations offers a number of theoretical advantages. The most obvious of these is a reduction in the amount of overlap between representations, thereby limiting interference between stored memories and allowing greater ease in decoding carried out by downstream projection regions (Perez-Orive et al. 2002). Indeed, our single neuron studies have shown that as faces are forgotten their sparse representation weakens and more generic encoding increases. Unsurprisingly, we have seen that performance in discriminating faces becomes progressively weaker when they are not viewed for a long time (Kendrick et al. 2001a).

\section{Theta-nested gamma oscillations and encoding}

An immediate question following on from discussions of population encoding for faces in temporal cortex is how such population based responses are co-ordinated across widespread cortical networks which may extend for several millimetres or more. Oscillatory rhythms present one mechanism for this and we have found not only significant theta $(4-8 \mathrm{~Hz})$ and gamma $(30-70 \mathrm{~Hz})$ activity in local field potential recordings but also evidence that these two frequencies are coupled (Kendrick et al. 2009 - theta phase and gamma amplitude) as has been reported in human neocortex (Canolty et al. 2006). Theta and gamma activities have been associated with various different aspects of cognitive functioning (Buzsaki et al. 2006) and with integration of visual feature processing (Csibra et al. 2000; Fries et al. 2001). Theta activity is remarkably synchronised across arrays of 64 electrodes $(\sim 2 \mathrm{~mm} \times 2 \mathrm{~mm})$ in both left and right temporal cortex and after face pair discriminations are learned the amplitude of theta during stimulus presentation increases significantly as does the theta-gamma ratio, theta and strength of coupling between theta and gamma and theta phase (Kendrick et al. 2009)(see Figure 6). These changes can occur in a matter of a few minutes as a learning criterion of $>80 \%$ correct is achieved during a single training session. Interestingly, simultaneous multiunit activity recordings revealed no overall significant changes in neuronal firing rates in the temporal cortex as a result of learning face or non-face object pair discriminations, thereby further supporting the possibility of a population based code being important.

We have developed a neural network model which reproduces these oscillatory rhythm and neuronal firing rate observations in the temporal cortex and where changes to glutamatergic NMDA receptor coupling are sufficient to reproduce effects of learning (Kendrick et al. 2009). This model predicts that as a result of the changes in theta-nested gamma there is a slight desynchronization in the firing of the temporal cortex output neurons and that this produces a significant potentiation of the firing of downstream projection neurons through a more effective 
temporal spread of the inputs reaching them. As predicted by the model there was indeed a significant learning-evoked desynchronization of temporal cortex neurons recorded across the 64 electrode arrays (Kendrick et al. 2009). Whether there is a corresponding facilitation of downstream responses in, for, example, the frontal cortex has yet to be confirmed.

\section{Conclusion}

The sheep brain clearly contains a broadly similar specialised face-processing system as in human and non-human primates and this allows remarkable accuracy in face identity and face emotion recognition for this highly social species. The strong evidence for right hemisphere dominance in face processing in sheep and findings of differences in processing faces by left and right temporal cortices may also help shed more light on why the human face-processing system is similarly lateralised. As such sheep represent an important additional animal model for studying the processing capacities of this complex, and socially important, neural system. Findings have already provided evidence for both sparse and population-based encoding and the presence and role of brain oscillatory rhythms in facilitating learning effects through widespread temporal changes in neuronal firing patterns. Future experiments will hopefully be able to establish more precisely the differential encoding of face identity and face emotion components and the respective roles of the left and right brain hemispheres and inform further developments in modelling how temporal cortex networks combine these sparse and population based codes. It is clear that temporal as well as spatial aspects of encoding will prove to be of key importance.

\section{References}

Alexander, G. and Shillito-Walser, E. (1977) Importance of visual cues from various body regions in maternal recognition of the young in Merino sheep (Ovis aries). Applied Animal Ethology, 3, 137-143.

Broad, K. D., Mimmack, M. L. and Kendrick, K. M. (2000) Is right hemisphere specialization for face discrimination specific to humans? European Journal of Neuroscience, 12, 731741.

Broad, K. D., Mimmack, M. L., Keverne E. B. and Kendrick, K. M. (2002) Increased BDNF and trk-B mRNA expression in cortical and limbic regions following formation of a social recognition memory. European Journal of Neuroscience, 16, 2166-2174.

Buszaki, G. (2006) Rhythms of the Brain. Oxford University Press, Oxford.

Canolty, R.T., Edwards, E., Dalal, S.S., Soltani, M., Nagarajan, S.S., Kirsch, H.E., Berger, M.S., Barbaro, N.M. and Knight R.T. (2006) High gamma power is phase-locked to theta oscillations in human neocortex. Science 313, 1626-1628.

Csibra, G., Davis, G., Spratling, M.W. and Johnson, M.H. (2000) Gamma Oscillations and Object Processing in the Infant Brain. Science 290, 1582-1585.

Da Costa, A.P., Leigh, A.E., Man, M-S. and Kendrick, K.M (2004) Face pictures reduce behavioural, autonomic, endocrine and neural indices of stress and fear in sheep. Proceedings of the Royal Society Biology B, 271, 2077-2084.

Elliker, K. (2006) Recognition of emotion in sheep. PhD Thesis, University of Cambridge.

Fabre-Nys, C., Ohkura, S. and Kendrick, K. M. (1997) Male faces and odours evoke differential patterns of neurochemical release in the mediobasal hypothalamus of the ewe during oestrus: an insight into sexual motivation? European Journal of Neuroscience, 9, 16661677.

Fries, P., Reynolds, J.H., Rorie, A.E. and Desimone, R.(2001) Modulation of Oscillatory Neuronal Synchronization by Selective Visual Attention. Science 291, 1560-1563. 
Freiwald, W. and Tsao, D. (2009) Taking apart the neural machinery of face processing. In: Handbook of Face Perception. Eds. A. Calder, G. Rhodes, J.V. Haxby and M. Johnson. Oxford University Press, Oxford. pp. xxxx-xxxxx

Kendrick, K.M. (2008) Sheep senses, social cognition and capacity for consciousness. In: The Welfare of Sheep. C. Dwyer ed. Springer, pp.135-158.

Kendrick, K. M. (1991) How the sheep's brain controls the visual recognition of animals and humans. Journal of Animal Science, 69, 5008-5016.

Kendrick, K.M. (1994) Neurobiological correlates of visual and olfactory recognition in sheep. Behavioral Processes, 33, 89-112.

Kendrick, K.M. (2006) Brain asymmetries for face recognition and emotion control in sheep. Cortex, 42, 96-98.

Kendrick, K. M., Atkins, K., Hinton, M. R., Broad, K. D., Fabre-Nys, C. and Keverne, B. (1995) Facial and vocal discrimination in sheep. Animal Behavour, 49, 1665-1676.

Kendrick, K. M., Atkins, K., Hinton, M. R., Heavens, P. and Keverne, B. (1996) Are Faces special for sheep? Evidence from facial and object discrimination learning tests showing effects of inversion and social familiarity. Behavioral Processes, 38, 19-35.

Kendrick, K.M. and Baldwin, B.A. (1987) Cells in temporal cortex of conscious sheep can respond to the sight of faces. Science, 236, 448-450.

Kendrick, K.M. and Baldwin, B.A. (1989) Visual responses of sheep temporal cortex cells to moving and stationary human images. Neuroscience Letters, 100, 193-197.

Kendrick, K.M., da Costa, A.P., Leigh, A.E., Hinton, M.R. and Peirce, J.W. (2001a) Sheep don't forget a face. Nature, 414,165-166.

Kendrick, K.M., Haupt, M.A., Hinton, M.R., Broad, K.D. \& Skinner, J.D. (2001b) Sex differences in the influence of mothers on the sociosexual preferences of their offspring. Hormones and Behavior, 40, 322-338.

Kendrick, K.M., Hinton, M.R., Atkins, K., Haupt, M.A. and Skinner, J.D. (1998) Mothers determine sexual preferences. Nature, 395, 229-230.

Kendrick, K.M., Leigh, A.E. and Peirce J. (2001c) Behavioural and neural correlates of mental imagery in sheep using face recognition paradigms. Animal Welfare 10, S89-101.

Kendrick, K.M., Zhan, Y., Fischer, H., Nicol, A.U., Zhang, X. and Feng, J. (2009) Learning alters theta-nested gamma oscillations in inferotemporal cortex. Nature Precedings (available online) and Nature Neuroscience (submitted)

Ladroue, C., Guo, S., Kendrick, K.M. and Feng, J. Beyond element-wise interactions: defining group-to-group interactions for biological processes. PLoS ONE, (submitted).

Leopold, D.A., Bondar, I.V. and Giese, M.A. (2006) Norm-based face encoding by single neurons in the monkey inferotemporal cortex. Nature, 442, 572-575.

O’Craven, K.M. and Kanwisher, N. (2000) Mental imagery of faces and places activates corresponding stimulus-specific brain regions. Journal of Cognitive Neuroscience, 12, 1013-1023.

Ohkura, S., Fabre-Nys, C., Broad, K.D. and Kendrick, K.M. (1997) Sex hormones enhance the impact of male sensory cues on both primary and association cortical components of visual and olfactory processing pathways as well as in limbic and hypothalamic regions in female sheep. Neuroscience, 80, 285-297.

Peirce, J.W. and Kendrick, K. M. (2002) Functional asymmetry in sheep temporal cortex. Neuroreport, 13, 2395-2399.

Peirce, J.W., Leigh, A.E. and Kendrick, K M. (2000). Configurational coding, familiarity and the right hemisphere advantage for face recognition in sheep. Neuropsychologia $\mathbf{3 8}$, 475-483.

Peirce, J.W., Leigh A.E., da Costa A.P.C. and Kendrick, K.M. (2001) Human face recognition in sheep: lack of configurational coding and right hemisphere advantage. Behavioral Processes, 55, 13-26. 
Perez-Orive, J., Mazor, O., Turner, G. C., Cassenaer, S., Wilson, R. I. and Laurent, G. (2002) Oscillations and sparsening of odor representations in the mushroom body. Science, 297, 359-65.

Perrett, D. I., Mistlin, A. J., Chitty, A. J., Smith, P. A., Potter, D. D., Broennimann, R. and Harries, M. (1988) Specialized face processing and hemispheric asymmetry in man and monkey: evidence from single unit and reaction time studies. Behavioral Brain Research, 29, 245-58.

Perrett, D. I., Oram, M. W., Harries, M. H., Bevan, R., Hietanen, J. K., Benson, P. J. and Thomas, S. (1991) Viewer-centred and object-centred coding of heads in the macaque temporal cortex. Experimental Brain Research 86,159-73.

Peters, M. and Jaspers-Fayer (2002) A laboratory manual for the dissection of the sheep brain. The University of Guelph.

Rolls, E.T., Treves, A. and Tovee, M.J. (1997) The representational capacity of the distributed encoding of information provided by populations of neurons in the primate temporal visual temporal cortex. Experimental Brain Research, 114, 149-162.

Quian Quiroga, R., Reddy, L., Kreiman, C., Koch, C. and Fried, I. (2005) Invariant visual representation by single neurons in the human brain. Nature, 435,1102-1107.

Tate, A.J., Fischer H., Leigh, A.E. and Kendrick, K.M. (2006) Behavioural and neurophysiological evidence for face emotion and face identity processing in animals. Philosophical Transactions of the Royal Society B, 361, 2187-2198.

Truett, A., Puce, A, Spencer, D.D. and McCarthy, G. (1999) Electrophysiological studies of human face perception. I: Potentials generated in occipitotemporal cortex by face and non-face stimuli. Cerebral Cortex, 9, 415-430. 


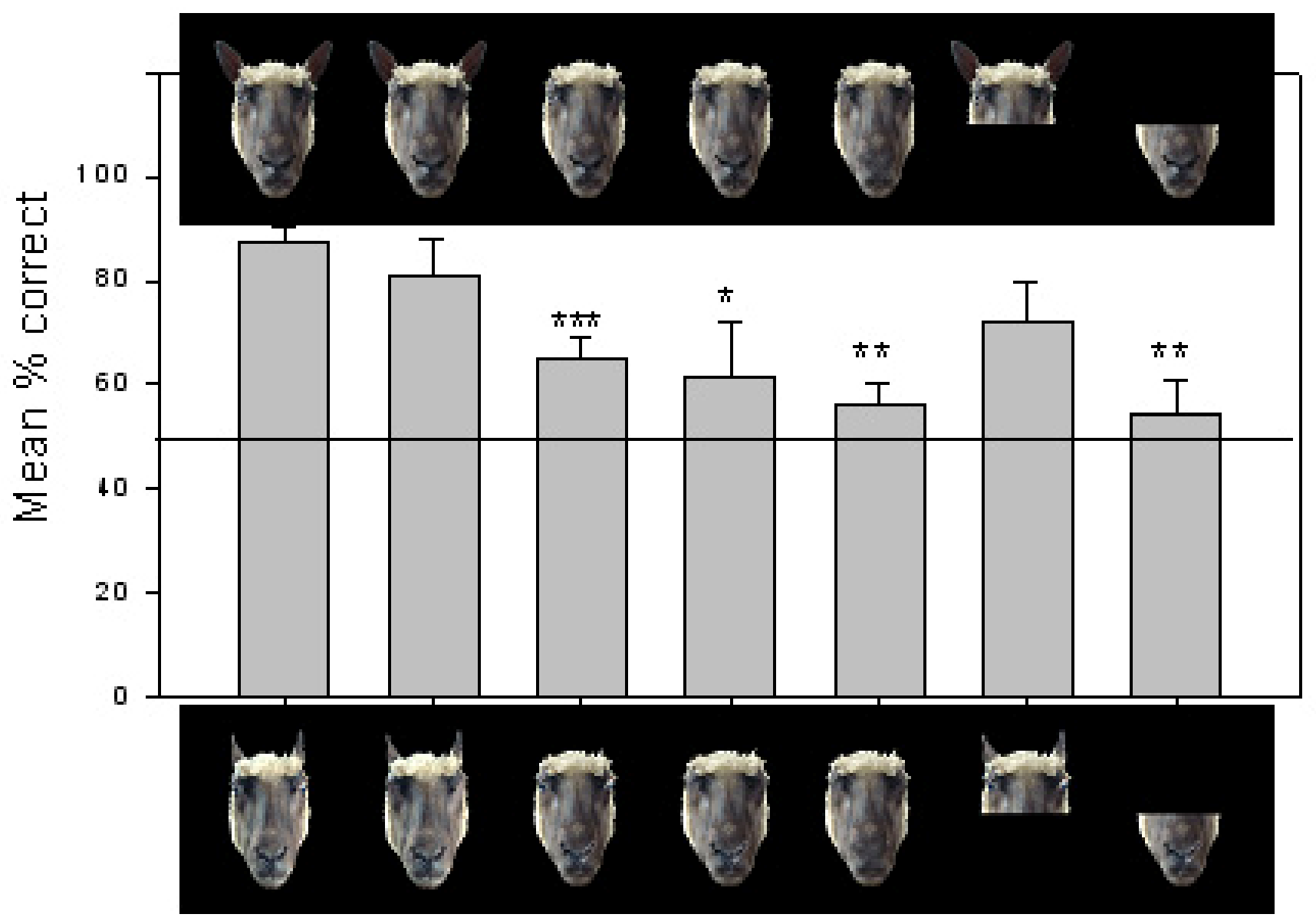

Figure 1. Mean \pm sem correct choice of a calm (bottom) vs stressed/anxious (top) version of the same sheep's face by 5 sheep following various manipulations of the face images (the first pair of images on the left are the control unaltered pictures). To avoid any possibility of the animals using subtle differences in face appearance to perform this discrimination, rather than emotion cues, the same version of the calm face was used in each case with alterations in ears, eyes, nose and mouth cause by stress/anxiety being substituted on calm face picture. ${ }^{* * *} \mathrm{P}<0.001$, $* * \mathrm{P}<0.01, * \mathrm{P}<0.05$ compared with the unaltered pictures (left). 

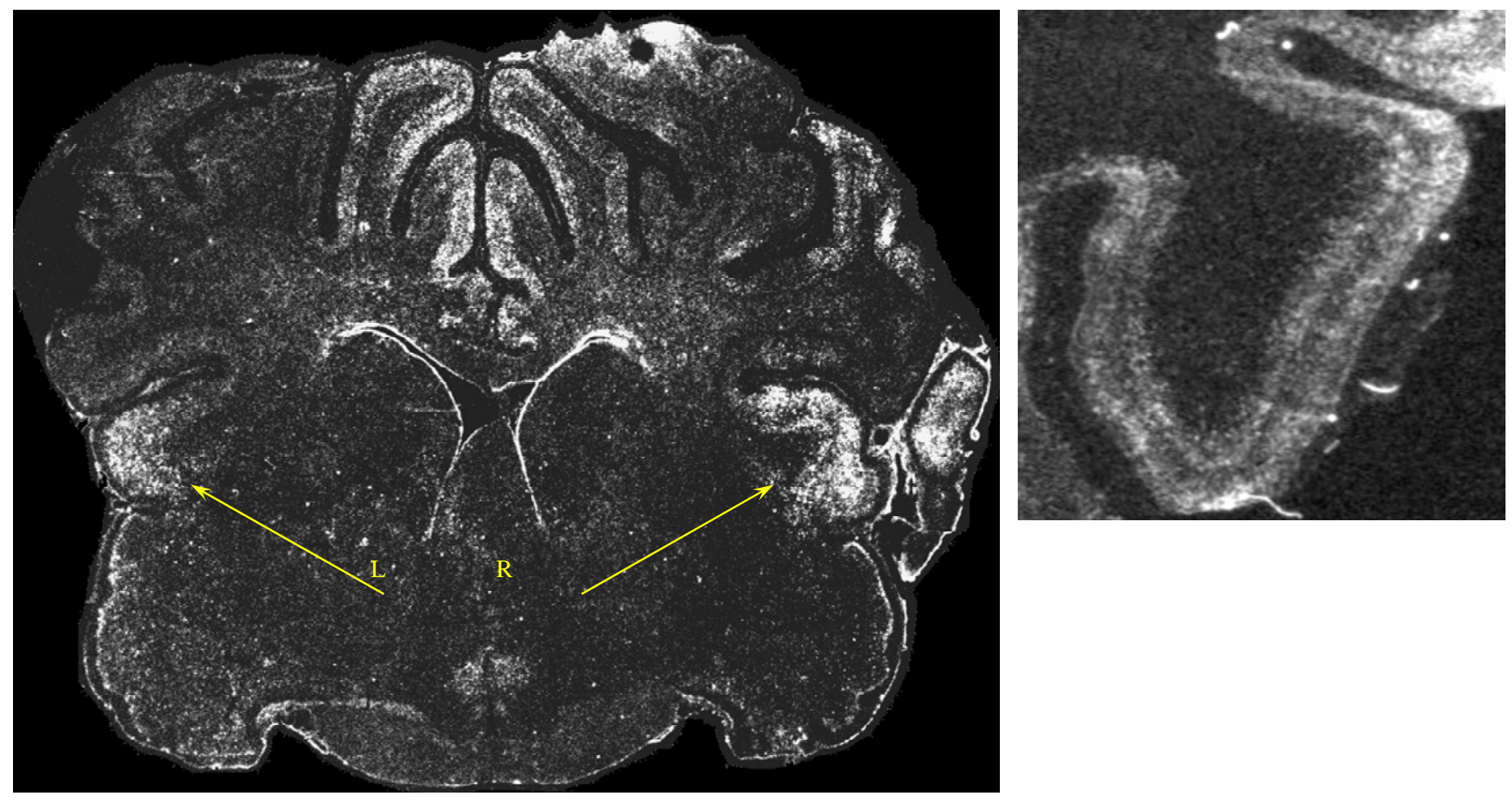

Figure 2. Dark-field autoradiograms showing on the left, increased c-fos mRNA expression (white areas) in the left and right superior and inferior temporal cortex of sheep after discriminating between two faces. Expression levels are much stronger in the right hemisphere and there is some suggestion of patches. On the right there is a higher magnification autoradiogram showing increased zif/268 mRNA expression in the right temporal cortex following exposure to a single sheep face stimulus. 
Faces
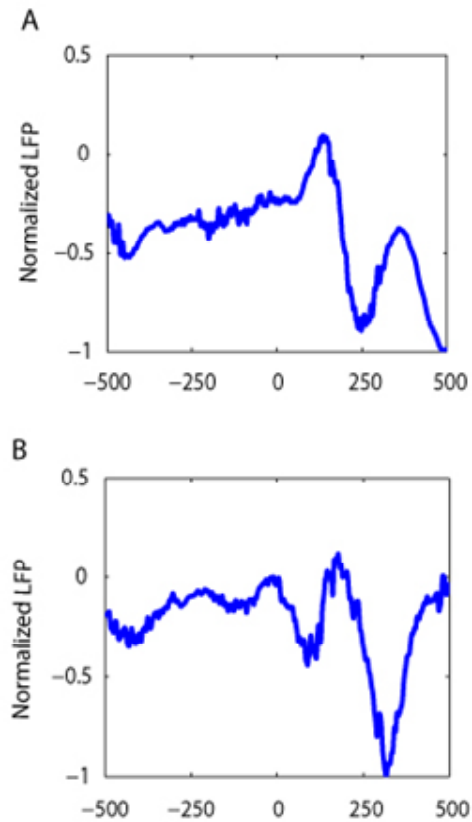

Inverted faces
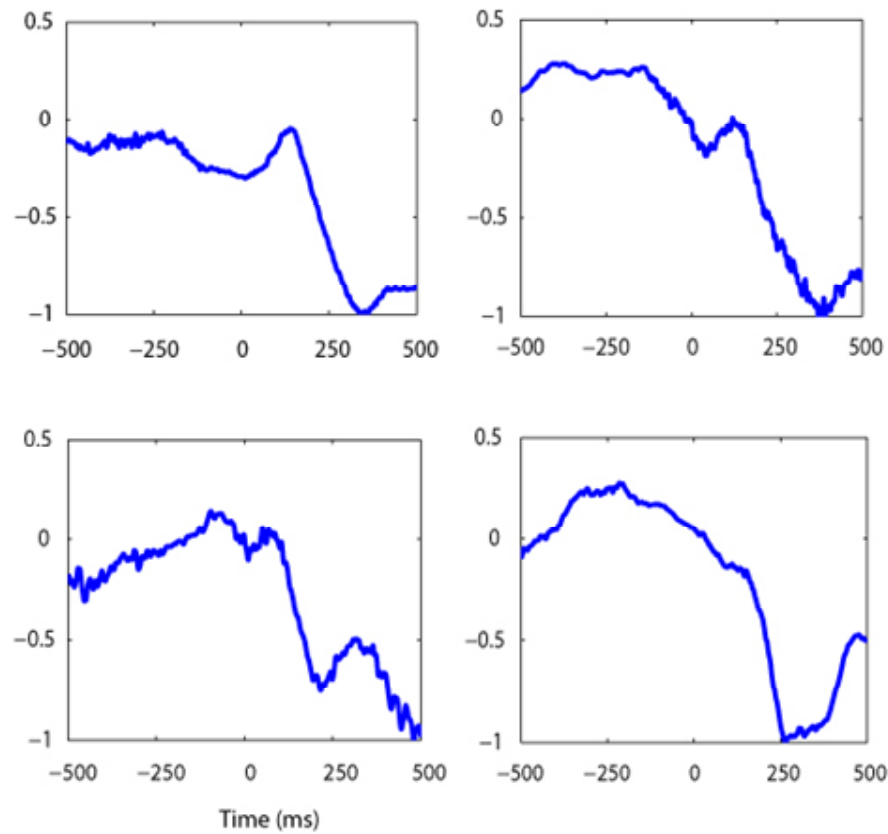

Figure 3. Local field potential recordings from 64 electrode arrays in the right temporal cortex of two sheep (A and B) showing average visual evoked potentials during exposure to paired face, inverted face or non-face objects (gloves vs phones or bucket vs bin). For both sheep data are from the same face pairs presented normally or inverted and for 40-60 trials). Sheep A: faces 4 vs 15 and sheep B faces 1721 vs 1724). The LFPs are presented normalised to allow easier comparison. Normally presented faces clearly evoke a more profound response particularly in the P120 component. 

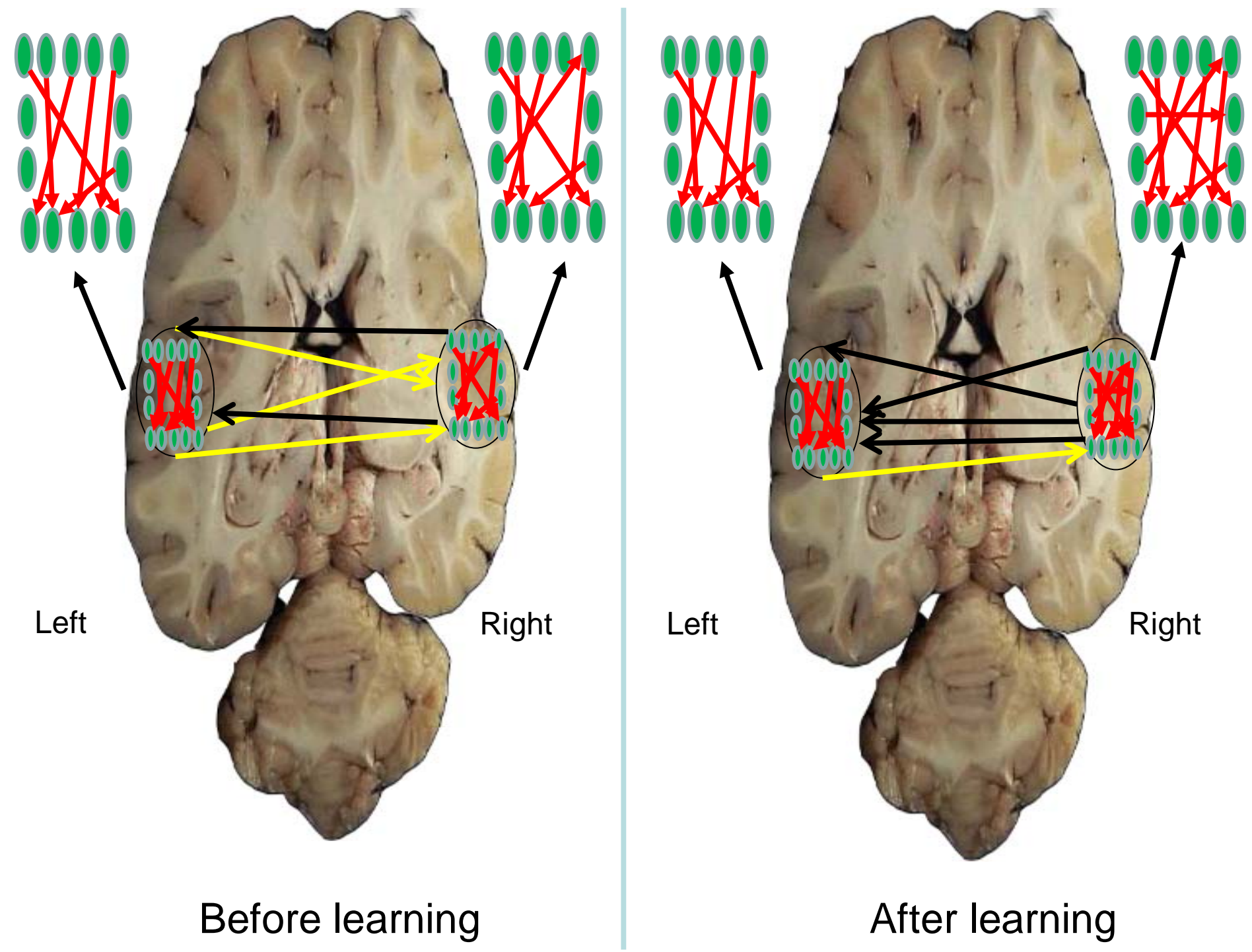

\section{After learning}

Figure 4. Significant causal connections within and between left and right temporal cortex of a sheep using partial Granger causality on local field potential data (Ladroue et al. submitted). The figure shows causal connectivity between 14 electrodes in each hemisphere and illustrates that the number increases within the right, but not the left hemisphere immediately successful ( $>80 \%$ correct) discrimination learning of a face pair has been achieved (data are from 20 trials when the animal only achieved 70\% correct - before learning - and the subsequent 20 trials when it achieved 95\% correct - after learning). There is also a shift from more causal connection from left to right (yellow arrows) before learning to more from right to left (black arrows) after it. These changes were maintained in subsequent trials after learning had been established and were present when causality was calculated for all 60 electrodes used. Horizontal brain section adapted from Peters and Jaspers-Fayer 2002. 

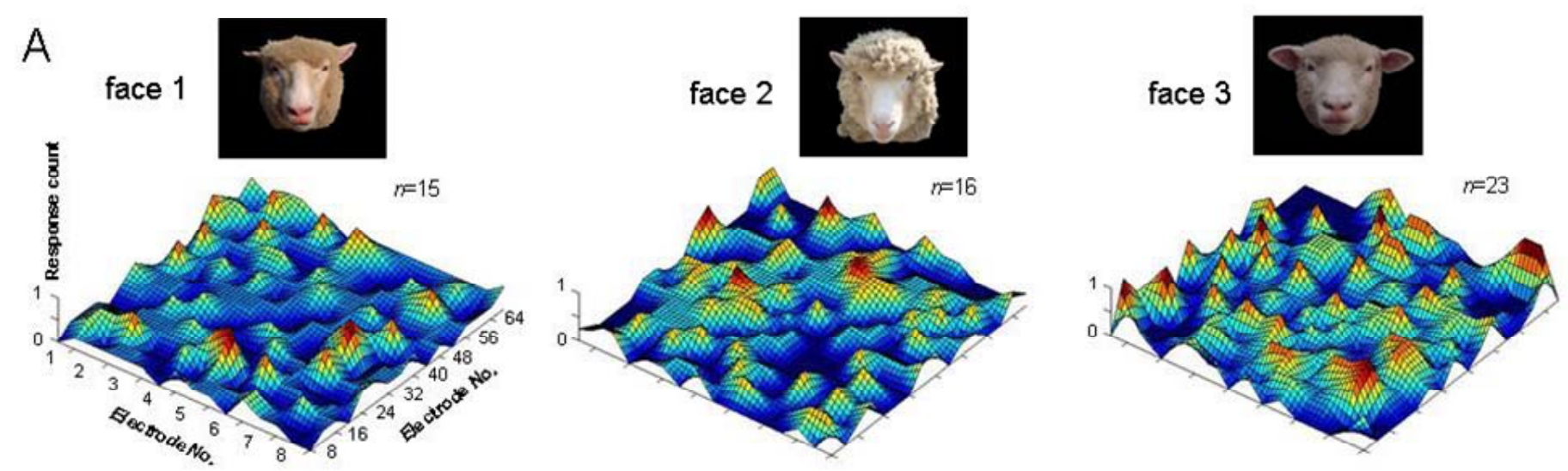

B

face $2 \longrightarrow 1$

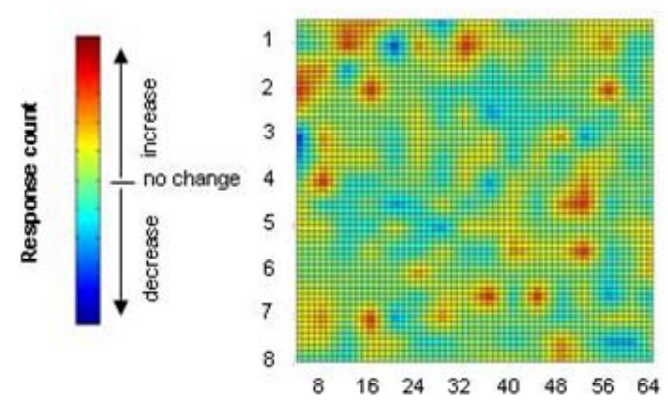

face $3 \rightarrow 2$

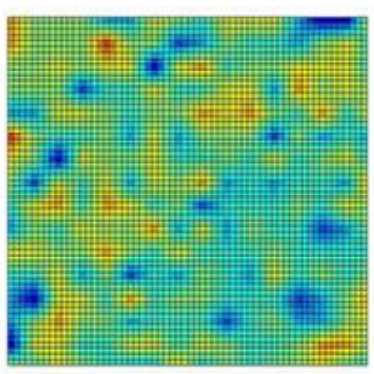

face $3 \longrightarrow 1$

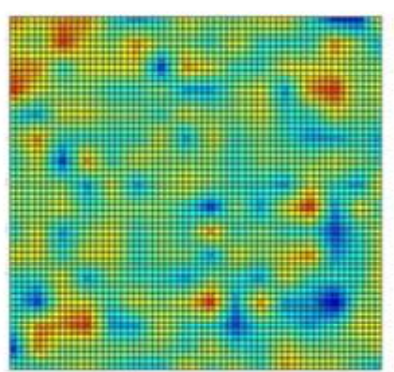

Figure 5. A. Pseudocolor 3D representations of averaged activity changes in single neurons in the right temporal cortex recorded by an $8 \times 8$ linear array of electrodes during correct discrimination of three different face pairs. Up to four different single neurons were recorded at each electrode. The pictures show the face fthat was rewarded. B. Maps showing differential activity changes between the three different face stimuli. 
A

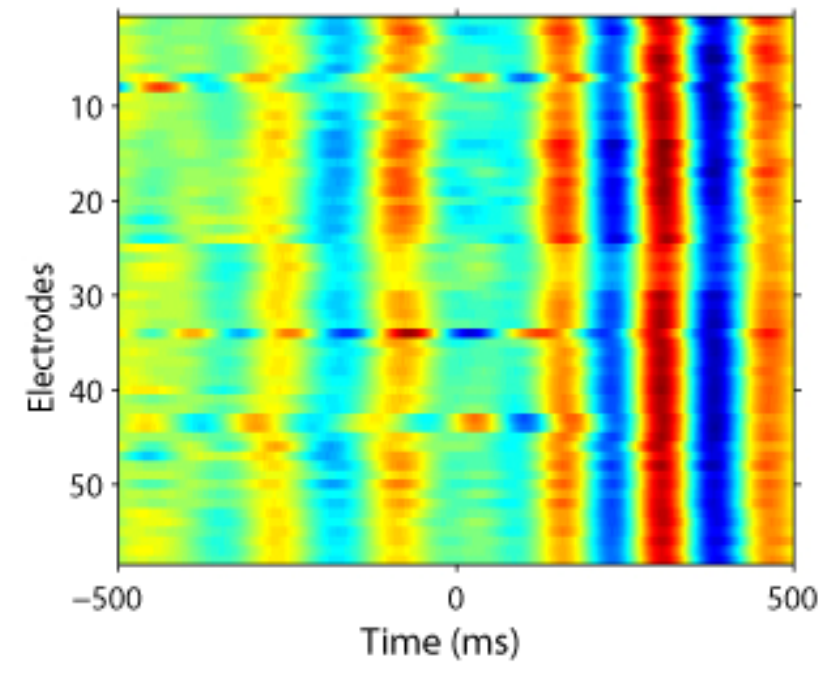

C

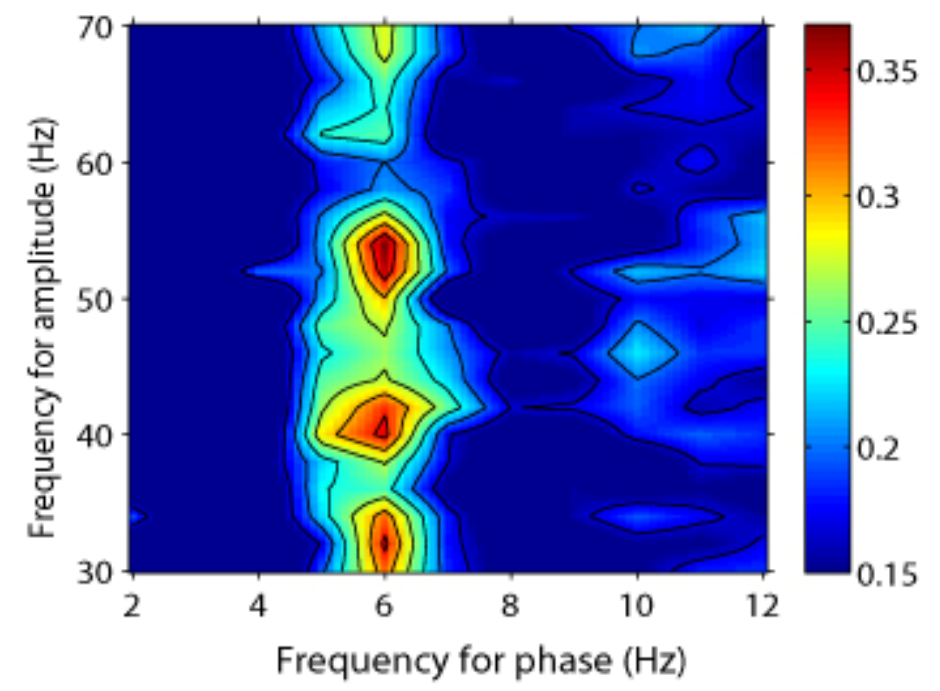

B

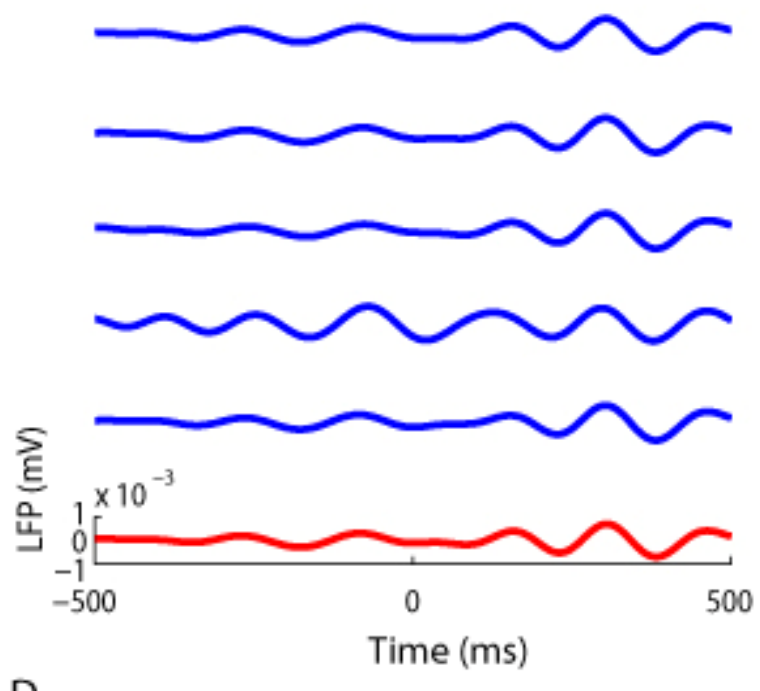

D

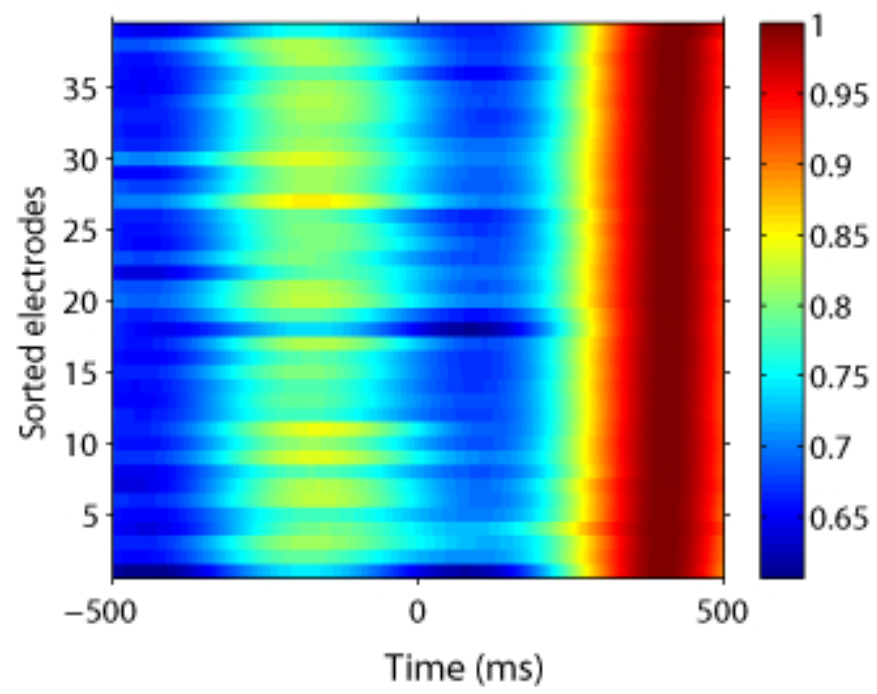

Figure 6. A: Pseudocolour representation of theta power across 58 recording electrodes in the right temporal cortex showing strong synchronisation across the array. The face pair stimulus is presented at time 0 . B. Shows the filtered (4-8Hz) local field potential (LFP) for 5 of the electrodes (blue) and an overall mean across all electrodes (red). C. Shows strong coherence across the array between theta phase (particularly 5-7 Hz - X-axis) and gamma amplitude (across the $30-70 \mathrm{~Hz}$ frequency range - y-axis) after learning a face pair discrimination. D. Shows a significant increase in theta amplitude in 40 of the electrodes following learning starting around $300 \mathrm{~ms}$ after the face-pair is presented at time 0 . 